\title{
MOTIVATIONAL FACTORS AND USER AWARENESS IN PATIENTS USING SKIN LIGHTENING CREAMS REPORTING TO DERMATOLOGY OUT PATIENT DEPARTMENT IN COMBINED MILITARY HOSPITAL RAWALPINDI
}

\author{
Sakina Sadiq Malik, Asher Ahmed Mashhood, Moizza Tahir*, Irfan Jamil Minhas, Sunniya Sadiq* \\ Combined Military Hospital/National University of Medical Sciences (NUMS) Rawalpindi Pakistan, *Combined Military Hospital Gujranwala/National \\ University of Medical Sciences (NUMS) Pakistan, **HQ Log 4 Corp, Lahore Pakistan
}

\begin{abstract}
Objective: To check the motivational factors behind the usage of skin lightening creams and users' awareness in our setup by constructing a validated questionnaire.

Study Design: Cross-sectional study.

Place and Duration of Study: Outpatient Department of Dermatology, Combined Military Hospital Rawalpindi, from Oct to Nov 2020.

Methodology: Two fifty patients who were using skin-lightening creams (SLC) were recruited after the informed consent. Questionnaire was made following seven steps as mentioned in AMEE (Association for Medical Education Europe) guide 87. Data was analyzed by SPSS version 21. Reliability was be checked by Cronbach@. Descriptive statistics of responses including frequencies and percentages were calculated. Exploratory factor analysis of questionnaire was made.

Results: Skin lightening cream users had age range from 16-56 years, majority 190 (76\%) were females. Out of 158 (63.2\%) were married, 180 (72\%) well educated and 146 (58.4\%) had Fitzpatrick skin type 3-5 predominate. Cronbach alpha of our questionnaire was 0.802 . The 5-point likert scale was used to record users' perceptions in the domains i.e., motivational factors, awareness about product and intentions. Majority, 89 (35.6\%) used the product on social media recommendation. Out of 201 (80.4\%) developed side effects with telangiectasia and skin thinning being the commonest ones. Out of 88 (35.2\%) were using other skin whitening practices besides these creams.

Conclusion: False perceptions about fairness and lack of user's awareness govern the skin lightening practices in our society resulting in increased usage and side effects experienced by users. A mass educational awareness program regarding "skin lightening creams" is the need of time.
\end{abstract}

Keywords: Motivational factors, Skin lightening creams, User awareness.

How to Cite This Article: Malik SS, Mashhood AA, Tahir M, Minhas IJ, Sadiq S. Motivational Factors and User Awareness in Patients Using Skin Lightening Creams Reporting to Dermatology out Patient Department in Combined Military Hospital Rawalpindi. Pak Armed Forces Med J 2021; 71(6): 1925-1929. Doi: $\quad$ Dttps://doi.org/10.51253/pafmj.v6i6.5680

This is an Open Access article distributed under the terms of the Creative Commons Attribution License (https://creativecommons.org/licenses/by-nc/4.0/), which permits unrestricted use, distribution, and reproduction in any medium, provided the original work is properly cited.

\section{INTRODUCTION}

Fair and flawless skin is the prime factor in defining beauty standards in our region where Fitzpatrick skin type 3-5 are predominate. Studies have shown that obsession for light complexion has significant positive relationship with the inferiority complex, social pressure and advertising. ${ }^{1}$ Marginalized darker individuals strive to get lighter skin color throughout their lives aiming at better social acceptability, success in career and economical privileges in both genders. The female appearance is considered a contested space for expression of social capital under influence of globalized beauty standards relying on Western ideals predominantly. ${ }^{2}$ Historically Korean women have been applying miansoo lotion and dregs of honey, Japanese applying white powder to the face, Pakistani and India

Correspondence: Dr Sakina Sadiq Malik, Consultant Dermatology, Combined Military Hospital Rawalpindi Pakistan

Received: 12 Nov 2020; revision received: 21 Jan 2021; accepted: 27 Jan 2021 ladies bath with turmeric, apply Ubtan and Multani Mitti and some Chinese women used to swallow powdered pearls in the hopes of becoming whiter. ${ }^{3}$ In Iran 79 plants have been identified as traditional herbal remedies of hyperpigmentation. ${ }^{4}$ Nowadays different lightening creams and serums, bleaching products, non-invasive cosmetic procedures performed by beauticians and aestheticians and even expensive aesthetic treatments by dermatologists are commonly used for achieving fairness.

"Colorism" and "skinderella" stories are being promoted by social media, highlighting fairness creams as the only way of achieving attractiveness and social success even for men. ${ }^{5}$ Half of all expenditure in the skincare industry is done on fairness creams, reflecting the relentless alarming prioritization of these products. ${ }^{6}$

Studies have revealed that many of these commercially available popular creams are contaminated with 
high toxic levels of mercury, lead, cadmium, copper and other heavy metals. ${ }^{7,8}$ According to a study in 2016 in Pakistan, salicylic acid is present in $87.88 \%$, Arbutin in $96.97 \%$, Cortisone in $60.60 \%$, Hydrocortisone in $48.48 \%$, Betamethasone valerate in $15.15 \%$ and Beta methasone dipropionate is present in $12.12 \%$ cosmetic creams available locally. ${ }^{9}$ Inadvertent usage of these creams containing toxic ingredients and corticosteroids lead to various local adverse effects like persistent facial erythema, aggravation of existing condition, addiction to product, hypertrichosis, telangiectasias, acneiform eruptions, rosacea and dyspigmentation. ${ }^{6,7}$ Patients using these creams during pregnancy are prone to have complications like neurological birth defects in children due to high mercury content of most creams. ${ }^{10}$

The objectives were to highlight the motivational factors leading to increased usage of SLCs, to find out about their use's awareness about the product and to find out the frequency of cutaneous side effects related to usage of SLCs in our patients. This research will help dermatologists to tailor an awareness program about the hazards of chronic usage of over the counter SLCs for our patients as guided by this study.

\section{METHODOLOGY}

It was a cross-sectional study was conducted at Outpatient Department of Dermatology, Combined Military Hospital Rawalpindi Pakistan, from October to November 2020, after permission from IRB CMH Rawalpindi: Ref. no. of IRC/ERB certificate 116/10/20.

We took reference prevalence from a Lahorebased study that revealed usage of SLCs was prevalent in $59 \%$ of female university students and staff. 7 With $95 \%$ significance level and power of 80 , sample size was calculated to be 28 initially by Epi-info sample size calculator. Since we were doing reliability analysis of a questionnaire, rules-of-thumb vary from four to 10 subjects per variable, with a minimum number of 190 subjects to ensure stability of the variance- covariance matrix to fill the questionnaire. ${ }^{11}$ We took a sample size of 250 finally to increase the reliability and precision of our results.

Inclusion Criteria: Patients (both male and female) reporting to OPD, who were using skin-lightening creams (SLCs) were included in the study.

Exclusion Criteria: Patients not consenting for the study were excluded.

Daily 60-70 patients were interrogated at random from OPD about usage of SLCs and positive respon- dents were asked to fill the questionnaire. About 250 patients were recruited after their consent. All patients were examined by dermatologists and their Fitzpatrick skin type was determined and mentioned on the questionnaire as well. ${ }^{12} \mathrm{~A}$ dermatology resident briefed them about the questionnaire and answered to their queries. A structured interview was conducted to cover the patterns of usage, side effects and miscellaneous aspects of SLC usage.

Questionnaire used in this study consisted of 10 questions covering 3 domains of users perceptions about SLCs i.e. motivation for fairness, awareness about SLCs usage and intentions. Responses were graded on a 5- point Likert scale ranging from strongly agree, agree, neutral, disagree and strongly disagree, It was constructed following 7 steps as mentioned in AMEE guide 87 for developing a questionnaire i.e., conducting a literature review for construct definition and determining if measures of construct already exists; interviewing 5 patients regarding their opinions about construct; synthesizing the literature review and interviews; developing a set of clear items and selecting the response options; conducting expert validation by getting it critically reviewed by fellow dermatologists; and conducting cognitive interviews. The seventh step i.e. pilot testing was done initially to check the reliability of the overall construct. ${ }^{13}$ It was tested initially on 10 subjects and value of Cronbach a of 0.85 indicated good internal consistency. It was named SLcIP (Skin lightening creams index of perceptions).

Quantitative data was analyzed by Statistical Package for the social sciences (SPSS) version 23. Reliability was checked by Cronbach a. Exploratory factor analysis was done to check the domains covered by questionnaire. Descriptive analysis was used for socio-demographics and other aspects of SLCs usage. Categorical data was summarized in tables showing frequencies and percentages.

\section{RESULTS}

Total 1480 patients were asked about usage of whitening creams. Out of them 250 patients (18.8\%) gave a positive response. Minimum age of users was 16 and maximum was 56 years with mean age of $33 \pm$ 8.97 years. Minimum age of starting the usage of skin lightening creams was 16 years and maximum was 55 years with mean of $31 \pm 8.12$ years. Sixty (24\%) were males and 190 (76\%) were females. Out of $158(63.2 \%)$ were married, $75(30 \%)$ were unmarried, $12(4.8 \%)$ were divorced and $5(2 \%)$ were widowed. $14(5.6 \%)$ were either not educated or received education below 
tenth grade, $56(22.4 \%)$ had education till tenth standard and $180(72 \%)$ were educated more than matric. 9 (3.6\%) had Fitzpatrick skin 1 or 2, $146(58.4 \%)$ had skin type 3 or 4 and 95 (38\%) had skin type 5 or 6 . Perceptions of users was recorded in the domains i.e. positive image of fair complexion and user awareness regarding whitening creams; by a 10 questions Likert scale response questionnaire. Reliability of this was calculated as Cronbach a of 0.802. The results are depicted in Figure-1.

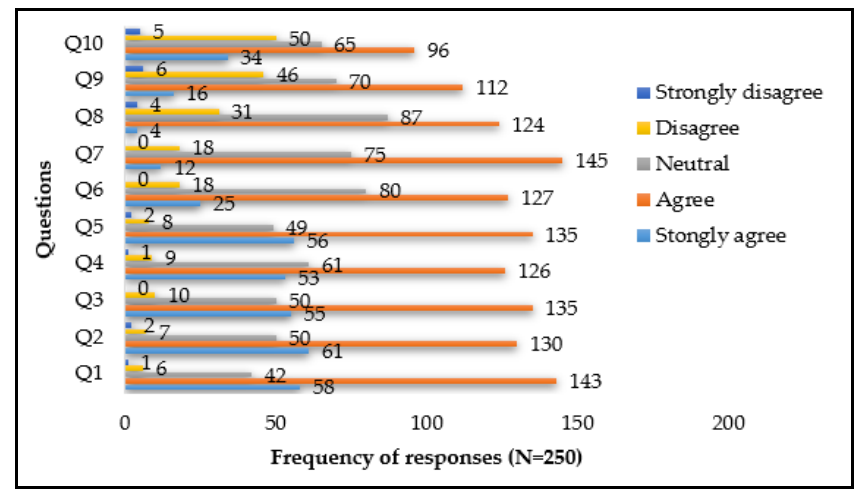

Figure-1: Frequencies of responses to SLcIP (Skin lightening creams index of perceptions).

Exploratory factor analysis EFA using the principal components method was computed on data obtained from 250 participants for these 10 questions (Table-I).

Table-I: Principal component analysis with rotated component matrix revealing component structure

\begin{tabular}{c|c|c|c}
\hline \multirow{2}{*}{ Questions } & \multicolumn{3}{|c}{ Components } \\
\cline { 2 - 4 } & 1-Motivation & $\begin{array}{c}\text { 2-Unawareness about } \\
\text { product }\end{array}$ & 3-Intentions \\
\hline 1 & 0.906 & & \\
\hline 5 & 0.890 & & \\
\hline 4 & 0.887 & & \\
\hline 2 & 0.835 & & \\
\hline 3 & 0.748 & & \\
\hline 6 & 0.701 & & 0.792 \\
\hline 9 & & 0.762 & \\
\hline 10 & & 0.699 & \\
\hline 8 & & & \\
\hline 7 & & & \\
\hline
\end{tabular}

None of the items were below the cut off criteria of 0.5. KMO was 0.810 showing sample adequacy. Bartlett's test of sphericity showed significance. All the communalities after extraction were above 0.5 . The scree plot (Figure-2) revealed eigenvalue of more than 1 for first three components and there after the curve tailed off gradually. Direct oblimin rotation yielded 3 stable factors i.e. motivational factors, user's unawareness about product and intentions, showing oblique rotation and correlation amongst data.

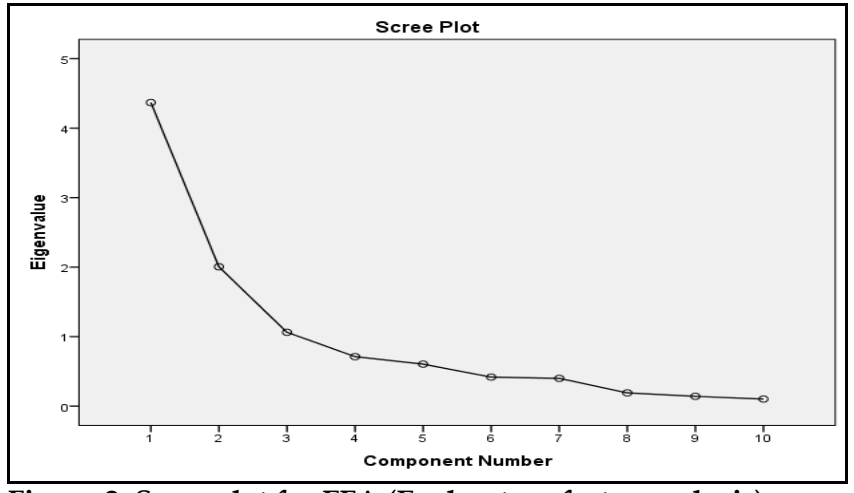

Figure-2: Scree plot for EFA (Exploratory factor analysis).

Patterns of cream usage, reason and influence for usage, improvement in complexion and longevity of results, side effects, sunscreen usage, no. of brands of lightening creams used, other skin lightening practices used and monthly expenditure on this; has been summarized in Table-II.

\section{DISCUSSION}

In our study, females were using skin-lightening creams three times more than males (76\% females: $24 \%$ males). It was much less than that in Jordan and Saudi Arabia where it was reported by $60.7 \%$ and $63.1 \%$ of the female respondents respectively. ${ }^{14,15}$ Similarly $35 \%$ of survey population was using bleaching creams in Kigali, Rwanda. ${ }^{16}$ Out of 600 South African women of dark skin, $32.7 \%$ reported using skin lightening products. ${ }^{17} 60.6 \%$ of female students were using these creams in a Malaysian survey. 18

Age of users ranged from 16-56 years with in our study, while age range was 18-70 years in a study carried out in South Africa where majority of African users were 20-30 years old and strangely majority of Indian users were above 50 years old. ${ }^{17}$

In a study in South African women $47 \%$ of users were married, $40 \%$ single, $4.8 \%$ divorced and $6 \%$ widowed which is comparable to our results of $63.2 \%$, $30 \%, 4.8 \%$ and $2 \%$ respectively without gender discrimination. ${ }^{17}$

Most of our users were educated i.e., $22.4 \%$ were matriculate and $72 \%$ more than matric, as compared to $30 \%$ and $59 \%$ respectively in another survey.$^{17}$ Interestingly $42 \%$ of female users were BSc degree holders in Jordan. ${ }^{14}$

$3.6 \%$ had Fitzpatrick skin 1 or 2, 58.4\% had skin type 3 or 4 and $38 \%$ had skin type 5 or 6 . Results are comparable to $36.5 \%$ light browns and $29 \%$ darker ones using these creams in South Africa and 126\% and $88 \%$ in Jordan respectively. ${ }^{17,14}$ 
Table-II: Patterns of skin lightening practices, side effects and Misc. data.

\begin{tabular}{|c|c|}
\hline Parameters & n (\%) \\
\hline \multicolumn{2}{|l|}{ Pattern of usage } \\
\hline Regularly & $133(53.2)$ \\
\hline Intermittently & $117(46.8)$ \\
\hline \multicolumn{2}{|l|}{ Recommending/influencing authority } \\
\hline Dermatologist & $40(16.0)$ \\
\hline Other doctor & $15(6.0)$ \\
\hline Beautician & $55(22.0)$ \\
\hline Social Media Ads & $89(35.6)$ \\
\hline Friends or Family & $51(20.4)$ \\
\hline \multicolumn{2}{|l|}{ Reason for usage } \\
\hline For fairness & $115(46.0)$ \\
\hline For hyper-pigmentary disorders & $135(54.0)$ \\
\hline \multicolumn{2}{|c|}{ Improvement in your complexion with usage } \\
\hline Yes & $218(87.2)$ \\
\hline No & $32(12.8)$ \\
\hline \multicolumn{2}{|l|}{ Long lasting improvement with usage } \\
\hline Yes & $107(42.8)$ \\
\hline No & $143(57.2)$ \\
\hline \multicolumn{2}{|l|}{ Side effects noticed } \\
\hline Nil & $49(19.6)$ \\
\hline Thinning of skin & $61(24.4)$ \\
\hline Telangiectasia & $71(28.4)$ \\
\hline Acne & $25(10.0)$ \\
\hline Redness/Irritation & $11(4.4)$ \\
\hline Dyspigmentation & $4(1.6)$ \\
\hline Hypertrichosis & $29(11.6)$ \\
\hline \multicolumn{2}{|l|}{ Usage of sunscreen } \\
\hline No & $62(24.8)$ \\
\hline Regularly & $91(36.4)$ \\
\hline Sometimes & $97(38.8)$ \\
\hline \multicolumn{2}{|c|}{ Brands of skin lightening creams used } \\
\hline Single & $146(58.4)$ \\
\hline More than one & $104(41.6)$ \\
\hline \multicolumn{2}{|c|}{ Other methods for skin lightening used } \\
\hline Nil & $162(64.8)$ \\
\hline Whitening pills & $26(10.4)$ \\
\hline Whitening injections & $11(4.4)$ \\
\hline Hydra facial/photo facial/Lasers & $35(14.0)$ \\
\hline Others or combination & $16(6.4)$ \\
\hline \multicolumn{2}{|c|}{ Monthly expenditure on skin lightening } \\
\hline Less than 2500Rs & $153(61.2)$ \\
\hline 2500-5000Rs & $37(14.8)$ \\
\hline More than 5000Rs & $60(24.0)$ \\
\hline
\end{tabular}

$35.6 \%$ of our users were influenced by social media for usage of these creams as compared to $77.4 \%$ women accepting advertisements majorly contribute to lightening cream usage. ${ }^{14} 22 \%$ of our respondents were advised these creams by doctors as compared to $3.2 \%$ in another survey and $20.4 \%$ by friends as compared to $51.9 \%$ in another study. ${ }^{17}$

$46 \%$ of our users used it for fairness as compared to $26.2 \%$ in South African survey and $18.5 \%$ in a local survey. $7,1729.3 \%$ ladies in another survey used hem for hyperpigmentary disorders. ${ }^{14} 36.4 \%$ were regularly using sunscreens in our respondents as compared with $21.4 \%$ sunscreen users in another survey. ${ }^{17}$

About $80.4 \%$ of our patients suffered from some side effects of these creams as compared to $34.9 \%$ of Malaysian females and $20 \%$ of North Indian population. ${ }^{18,19}$ In a local survey, facial erythema in $51.8 \%$ of subjects was most common adverse effect followed by aggravation of pre-existing skin condition in $49 \%$, steroid addiction in $32.5 \%$, and hypertrichosis in $26.5 \%$, telangiectasia in $18 \%$ and acne in $13.8 \%$ of patients. Our results varied with telangiectasia as most common side effect i.e., $28.4 \%$, skin thinning in $24.4 \%$, hypertrichosis in $11.6 \%$, acne in $10 \%$ and erythema in $4.4 \%$. These differences could be due to changes in composition of local available whitening creams.

$61.2 \%$ of our respondents were spending less than 2500 Rs monthly on skin lightening practices and $24 \%$ were spending more than 5000 Rs monthly. We spend more than South Africans where 39\% women spend less than 20 Rands on these practices and $7.4 \%$ spend more than 200 Rands monthly. ${ }^{1}$ Rand is equal to 9.81 PKR. ${ }^{17}$

The reliability of our questionnaire related to perceptions of users was 0.8 that is good reliability showing good internal consistency of items used in the questionnaire. ${ }^{20}$ EFA revealed three domains in our questionnaire which added "intentions" to our two initially thought domains i.e. motivational factors and user's unawareness.

Most of our patients agreed that as fair complexion is the key to social success, better career and is indeed a symbol of high social status. $52 \%$ of our respondents agreed with association of fairer skin with better marital outcomes which is comparable to $43 \%$ in a survey carried out in Jordan. 22\% strongly agreed and $54 \%$ agreed fairness gives better quality of life similar to $53.8 \%$ Malaysian females who associated it with increased self-esteem. ${ }^{18}$ They considered it beneficial for skin health without needing to know their ingredients as compared to $32 \%$ Malaysians females who preferred checking ingredients before buying these. ${ }^{18} 72.5 \%$ of Jordanian ladies did not have knowledge about ingredients of products. ${ }^{14}$ The denied need for consultation by dermatologist prior to usage and $52 \%$ were even willing to accept their side effects in return for their assumed benefits similar to $37.5 \%$ Jordanian ladies who did not believe in their side effects either. $62.8 \%$ wanted to recommend these creams to their near and dear ones as they were satisfied like $70.9 \%$ ladies in another survey. ${ }^{14}$ 
This study can be considered as a need assessment venture for designing a program for educating people regarding misuse of whitening creams and creating user awareness about their side effects and checking for safe ingredients in them. SLcIP questionnaire can be used in this regard especially.

\section{LIMITATION OF STUDY}

Study population was from dermatology OPD dealing a specific clientage, who were mostly educated, which is not an appropriate sample for generalization of results. Names of brands and their constituents were not mentioned which could have been effectively correlated with the side effects mentioned.

\section{CONCLUSION}

Large number of our study population were using skinwhitening creams either for fairness or hyperpigmentary disorders. Their positive perceptions about fairness and lack of user awareness about these creams govern their practices. They experience side effects but still use these products most guided by social media advertisements.

\section{Conflict of Interest: None.}

\section{Authors' Contribution}

SSM: Direct contibution conception, AAM: Contribution and collection of data, MT: Questionnaire development, IJM Questionnaire development, SS: Data Analysis.

\section{REFERENCES}

1. Ismail S, Loya S, Hussain J. Obsession for Fair Skin Color in Pakistan. Int J Innov Res Educ Sci 2015; 2(6): 2349-5219.

2. Rahbari L, Dierickx S, Longman C, Coene G. 'Kill Me but Make Me Beautiful': Harm and agency in female beauty practices in contemporary Iran. Iran Cauc. Year Of Study Missing 2013; 22(1):50-60.

3. Li EPH, Min HJ, Belk RW, Kimura J, Bahl S. Skin lightening and beauty in four Asian cultures. "Skin Lightening and Beauty in Four Asian Cultures", in NA - Advances in Consumer Research Volume 35, eds. Angela Y, Soman LD, Duluth MN: Association for Consumer Res 2008; 35(1): 444-449.

4. Ghafari S, Fahimi S, Sahranavard S. Plants used to treat hyperpigmentation in Iranian traditional medicine: a review. Res J Pharmacogn. 2017;4(4):71-85.

5. Mukherjee S. Darker shades of "fairness" in India: Male attractiveness and colorism in commercials. Open Linguist 2020; 6(1): 225-248.
6. Pollock S, Taylor S, Oyerinde O, Nurmohamed S, Dlova N, Sarkar R, et al. The dark side of skin lightening: an international collaboration and review of a public health issue affecting dermatology. Int J Womens Dermatol 2021; 7(2): 158-164.

7. Chohan SN, Suhail M, Salman S, Bajwa UM, Saeed M, Kausar S, et al. Facial abuse of topical steroids and fairness creams: A clinical study of 200 patients. J. Pak. Assoc. Dermatol 2014; 24(3): 204-211.

8. Peregrino CP, Moreno MV, Miranda SV, Rubio AD, Leal LO. Mercury levels in locally manufactured Mexican skin-lightening creams. Int J Environ Res Public Health 2011; 8(6): 2516-2523.

9. Shams A, Khan IU, Iqbal H. Analysis of salicylic acid, arbutin and corticosteroids in skin whitening creams available in Pakistan using chromatographic techniques. Int J Cosmet Sci 2016; 38(4): 421-428.

10. Al-Saleh I. Potential health consequences of applying mercurycontaining skin-lightening creams during pregnancy and lactation periods. Int J Hyg Environ Health 2016; 219(4): 468-474.

11. Terwee CB, Bot SDM, de Boer MR, van-der-Windt DAWM, Knol DL, Dekker J, et al. Quality criteria were proposed for measurement properties of health status questionnaires. J Clin Epidemiol 2007; 60(1): 34-42.

12. Med D. Objective determination of Fitzpatrick skin type. Dan Med Bull 2010; 57(8): 4153.

13. Artino AR, La Rochelle JS, Dezee KJ, Gehlbach H. Developing questionnaires for educational research: AMEE Guide No. 87. Med Teach 2014; 36(6): 463-474.

14. Hamed SH, Tayyem R, Nimer N, AlKhatib HS. Skin-lightening practice among women living in Jordan: Prevalence, determinants, and user's awareness. Int J Dermatol 2010; 49(4): 414-420.

15. Alrayyes SF, Alrayyes SF, Farooq Dar U. Skin-lightening practices behind the veil: An epidemiological study among Saudi women. J Cosmet Dermatol 2020; 19(1): 147-153.

16. Kamagaju L, Morandini R, Gahongayire F, Stévigny C, Ghanem G, Pirotte G, et al. Survey on skin-lightening practices and cosmetics in Kigali, Rwanda. Int J Dermatol 2016; 55(1): 45-51.

17. Dlova NC, Hamed SH, Tsoka-Gwegweni J, Grobler A. Skin lightening practices: An epidemiological study of South African women of African and Indian ancestries. Br J Dermatol 2015; 173(S2): 2-9.

18. Rusmadi SZ, Ismail SNS. Preliminary study on the skin lightening practice and health symptoms among female students in Malaysia. J Environ Public Health 2015; 2015(1): 591790.

19. Wong C, Wong S, Tang H, Minocha R, Singh R, Grills N. Use of skin-lightening products among outpatient attendees in a North Indian Hospital. Indian J. Public Health 2017; 61(2): 137-140.

20. George D, Mallery P. SPSS for Windows Step by Step: A simple guide and reference, 11.0 Update. 4th ed. Boston: Allyn \& Bacon.Chapter 18, Reliability analysis; 2003: p.53-55. 